\title{
The measure of existence of a quantum world and the Sleeping Beauty Problem
}

\author{
Berry Groisman ${ }^{\dagger \dagger}$, Na'ama Hallakoun* and Lev Vaidman* \\ $\uparrow$ Sidney Sussex College, Cambridge, CB2 3HU, UK \\ $\$$ DAMTP, University of Cambridge, CB3 0WA, UK \\ * School of Physics and Astronomy, Tel-Aviv University, 69978, Israel
}

\begin{abstract}
An attempt to resolve the controversy regarding the solution of the Sleeping Beauty Problem in the framework of the Many-Worlds Interpretation led to a new controversy regarding the Quantum Sleeping Beauty Problem. We apply the concept of a measure of existence of a world and reach the solution known as 'thirder' solution which differs from Peter Lewis's 'halfer' assertion. We argue that this method provides a simple and powerful tool for analysing rational decision theory problems.
\end{abstract}

\section{Quantum Sleeping Beauty controversy}

The Sleeping Beauty Problem (SBP) (Elga 2000, Lewis 2001) inflamed an ongoing controversy amongst researchers in rational Bayesian decision theory. One group claimed that in a certain coin toss scenario, Beauty must have credence one-third for Heads, while another group argued for one-half, with no clear consensus on a solution in sight (Pust 2011, Thorn 2011). Lev Vaidman (2001) proposed to consider the SBP within the framework of the Many-Worlds Interpretation of Quantum Mechanics (MWI). He argued that treating the issue of probability in the MWI using the concept of the measure of existence of a world, makes the SBP simpler, yielding the thirder solution. A few years later, Peter Lewis (2007) also suggested solving the 
SBP within the MWI framework. He, however, argued for the halfer solution. This prompted a response by David Papineau and Víctor Durà-Vilà (2009a), followed by an exchange of replies (Lewis 2009, Papineau and Durà-Vilà 2009b). Other authors made subsequent contributions to the controversy (Peterson 2011, Bradley 2011, Tappenden 2011, Wilson 2013) proposing some modified scenarios.

Peter Lewis introduced the Simplified SBP and put at the heart of his argument, its similarity to the Sleeping Pill Experiment (Vaidman 1998). Papineau and Durà-Vilà, the thirders, responded by questioning this similarity on metaphysical grounds. However, they argued that accepting this similarity and Vaidman's approach leads to the halfer solution. Yet, we argue that Vaidman's original suggestion of treating the SBP within the MWI framework using the concept of the measure of existence leads to a straightforward thirder solution, in spite of the strong similarity.

\section{From the measure of existence of a world to the illusion of probability}

In our view, there is no genuine probability in the MWI framework. It is a completely deterministic theory and there is no relevant information that an observer, preparing a quantum experiment, is ignorant about. The quantum state of the Universe at one time determines the quantum state at all times. Vaidman (1998) associated our everyday perception of probability with post-measurement ignorance. In his Sleeping Pill Experiment (SPE), the agent is given a sleeping pill and sleeps through a quantum measurement, which serves as a quantum coin toss. While asleep, she is moved to either room $H$ or to an identical-looking room $T$, based on the result of the toss - Heads or Tails. Upon awakening, she is asked what her credence in Heads is. It is meaningless to ask the agent before the toss what is the probability to find herself in room $H$ after the toss, since she is the ancestor of both future descendants. However, after the experiment, we can ask her descendant, in which room does she think she is. The fact that there is no direct meaning for the probability of the outcomes of the experiment, does not contradict a genuine uncertainty on the part of the two descendants upon awakening in the propositions 'I am in the $H$-world' and 'I am in the $T$-world'. 
The illusion of probability follows from the identity between the quantum state of the hypothetical Collapse Universe and the quantum state of the corresponding world in the MWI Universe. An observer in a Collapse Universe, performing a sequence of experiments, will have the same memories as an observer in a MWI-Universe in a world with corresponding results.

In the MWI, the squared absolute value of the amplitude of a world is called its 'measure of existence'. The 'behaviour principle' teaches us that one should care about one's descendants according to the measures of existence of their worlds, and thus functions as the Born Rule counterpart in the MWI. Paul Tappenden (2011) has named this the Born-Vaidman Rule. As it is stated in (Vaidman 1998): 'The relative measures of existence of the worlds into which the world splits provide the concept of probability'

In this paper, we accept the Born-Vaidman rule and show how it helps to analyse the SBP in a straightforward way. Note that an alternative approach which assigns a pre-branching uncertainty (Saunders and Wallace 2008), does not lead to a different conclusion. The analysis in that framework, however, does not benefit from the advantages of our deterministic approach.

\section{The Simplified Sleeping Beauty Problem versus the Sleeping Pill Experiment}

In Peter Lewis's Simplified SBP, Beauty goes to sleep on Sunday evening. The researchers wake her up twice - once on Monday and once on Tuesday. Upon each awakening, Beauty is asked about her degree of belief in the proposition 'This is the Monday awakening'. Beauty's task becomes non-trivial because she forgets the Monday awakening completely, due to a memory erasure pill given to her before she goes back to sleep. Lewis argued that Beauty's subjective experience is very similar to the experience of the agent awakened in one of the rooms in the SPE.

There is a strong case for the similarity advocated by Lewis. What unifies both scenarios is the fact that Beauty's subjective uncertainty takes place on the background of objective certainty. The researchers possess full information throughout the experiment and do not share Beauty's ignorance. The effect of the memory erasure pill in the simplified SBP makes Beauty's

experience identical to that of the agent in the SPE. Beauty ought to have the same degree of 
belief in the propositions 'This is the Monday awakening' as in the corresponding proposition 'I am in the $H$-world'. Henceforth we identify Beauty with both experiments.

In the Simplified SBP, there is only one branch with which we can therefore associate a measure of existence 1. It is the same for Monday and Tuesday, so Beauty should attribute equal credences to both options, which thus have to be $1 \frac{1}{2}$. In the SPE, the quantum state of the world upon Beauty's awakening is

$$
|\Psi\rangle=\frac{1}{\sqrt{2}}|H\rangle+\frac{1}{\sqrt{2}}|T\rangle,
$$

and the measure of existence of each branch is $1 / 2$. From the equality of the measures of existence it follows that Beauty should attribute credence $1 / 2$ again.

The measures of existence of the worlds upon Beauty awakenings are equal for the two alternatives in Lewis's case as well as in the SPE, but different between the two cases, see Figure 1. Like Papineau and Durà-Vilà we think that this is relevant for the solution, however, we disagree when they attribute certainty to the Monday and Tuesday awakenings, but credences of $1 / 2$ to the $H$ and $T$ results. They seem to confuse an external observer's and Beauty's points of view. An external observer cannot assign credence $1 / 2$ for Beauty being in $H$ or $T$ world. Only Beauty possesses credence of $1 / 2$ for these events, but she, upon awakening, also has credence $1 / 2$ for Monday and for Tuesday. 


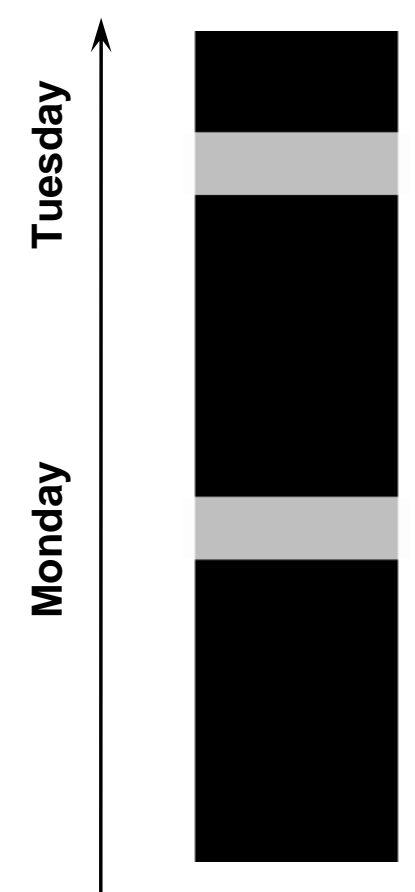

(a)

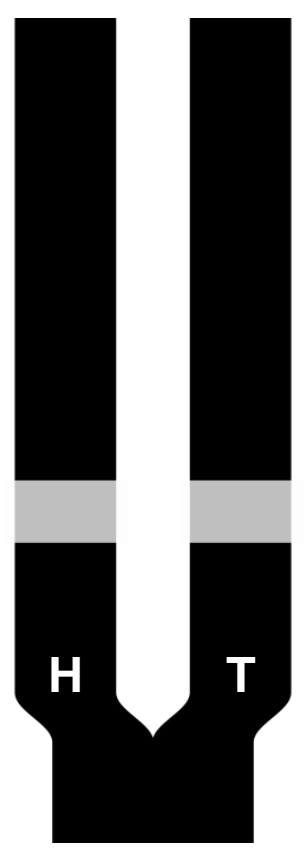

(b)

Figure 1: World lines in the Simplified Sleeping Beauty Problem $(a)$ and in the Sleeping Pill Experiment $(b)$. The width of the lines represents the measures of existence of corresponding worlds. The bright areas correspond to awakenings.

\section{The Quantum Sleeping Beauty Problem}

In the full SBP setup, Beauty goes to sleep on Sunday. She knows that a fair coin will be tossed while she is asleep. If the coin lands Tails, then as in the Simplified SBP, she will be awakened once on Monday and once on Tuesday, without having on Tuesday any memory of the previous awakening. If the coin lands Heads, then Beauty is awakened on Monday only. Upon each awakening, she is asked for her credence in the proposition 'The coin has landed Heads'. Adam Elga (2000) argued that her credence should be 1/3, while David Lewis (2001) argued for 1/2. Since then, philosophers have been divided between halfers and thirders. In an attempt to resolve the controversy, Vaidman (2001) proposed to use a quantum coin and to consider the problem within the MWI framework. He deduced the thirder solution. Peter Lewis (2007) also addressed the quantum SBP (QSBP) in the MWI framework. However, based on the similarity discussed in the previous section, he replaced the double awakening in the full SBP with a SPE, and deduced the halfer solution. 
Let us analyse the two scenarios using the concept of the measure of existence. We start with the analysis of the branches of the original full QSBP experiment. With an obvious notation, the wavefunction on Monday is

$$
\left.\left.|\Psi\rangle_{\text {Monday }}=\frac{1}{\sqrt{2}} \mid H \text {, 过 }\right\rangle_{\text {Monday }}+\frac{1}{\sqrt{2}} \mid T \text {, }\right\rangle_{\text {Monday }}
$$

and the wavefunction on Tuesday is

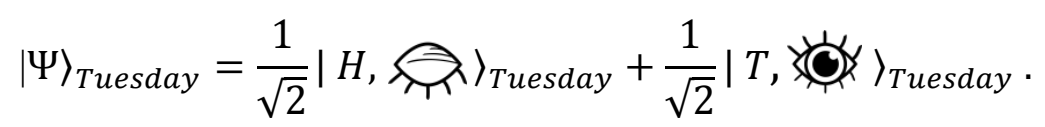

Upon awakening, Beauty knows that she is in one of three possible events: either it is Monday and the coin landed Heads $\left(H_{M o n}\right)$, or Monday and Tails $\left(T_{M o n}\right)$, or Tuesday and Tails $\left(T_{T u e}\right)$. The measures of existence of the corresponding branches are equal, see Figure 2(a). Since only one of the events corresponds to Heads, her credence in Heads should be (Vaidman 2001):

$$
\operatorname{Cr}(H)=\frac{\mu\left(H_{\text {Mon }}\right)}{\mu\left(H_{\text {Mon }}\right)+\mu\left(T_{\text {Mon }}\right)+\mu\left(T_{\text {Tue }}\right)}=\frac{\frac{1}{2}}{\frac{1}{2}+\frac{1}{2}+\frac{1}{2}}=\frac{1}{3},
$$

where $\mu(x)$ denotes the measure of existence of the branch with an event $x$. 


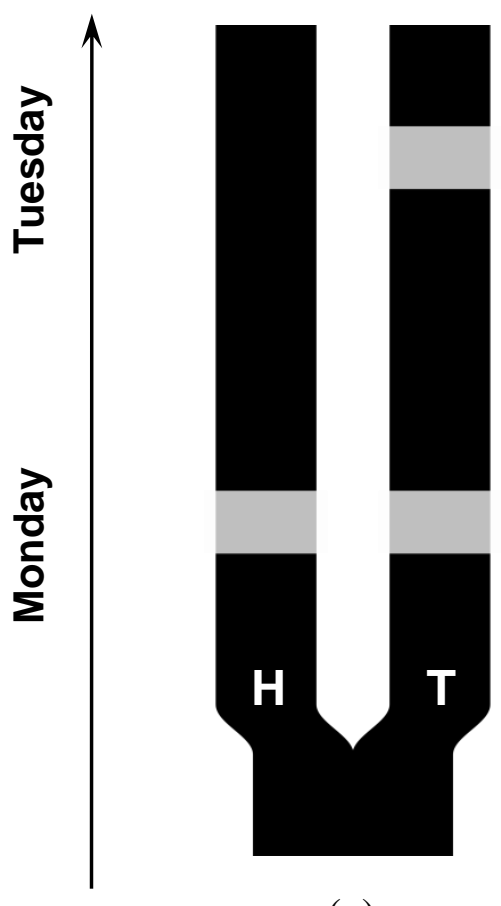

(a)

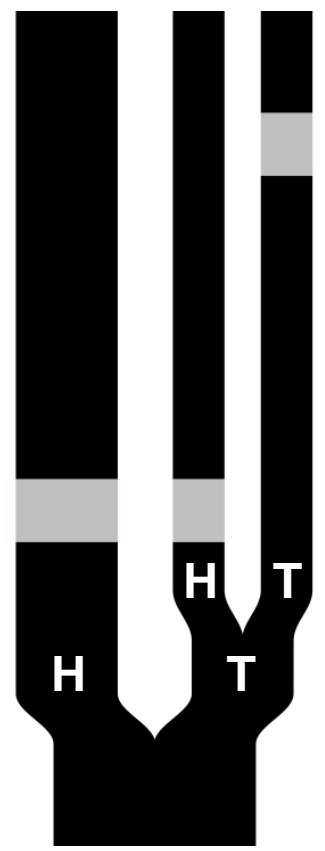

(b)

Figure 2: (a) World lines in the original Sleeping Beauty Problem. The measures of existence of worlds $H$ and $T$ are $1 / 2$. (b) World lines in Lewis's version of the Sleeping Beauty Problem. The measures of existence of the world $H$ is $1 / 2$ and of the worlds $T H$ and $T T$ are $1 / 4$. The bright areas correspond to awakenings.

In Lewis's version of the experiment, in the case of the Tails result, another coin is tossed. If the second coin lands Heads, Beauty is awaken only on Monday, and if Tails - only on Tuesday. The wavefunction on Monday is

$$
|\Psi\rangle_{\text {Monday }}=\frac{1}{\sqrt{2}}|H,\rangle_{\text {Monday }}+\frac{1}{2}|T H,\rangle_{\text {Monday }}+\frac{1}{2}\left|T T, \hat{\gamma}_{\lambda}\right\rangle_{\text {Monday }},
$$

and on Tuesday is

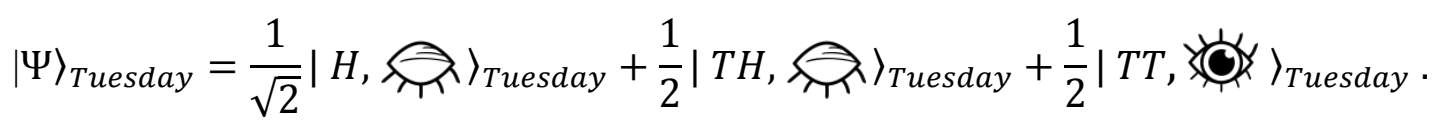

Again, upon awakening, Beauty knows that she is in one of three possible events corresponding to $H, T H$ and $T T$ results. However, the measures of existence of the branches are not equal: they are $1 / 2,1 / 4$ and $1 / 4$ respectively, see Figure 2(b). Therefore, in Lewis's double-coin experiment, Beauty should have credence $1 / 2$ in the first coin landing Heads: 


$$
\operatorname{Cr}(H)=\frac{\mu\left(H_{\text {Mon }}\right)}{\mu\left(H_{\text {Mon }}\right)+\mu\left(T H_{\text {Mon }}\right)+\mu\left(T T_{\text {Tue }}\right)}=\frac{\frac{1}{2}}{\frac{1}{2}+\frac{1}{4}+\frac{1}{4}}=\frac{1}{2} .
$$

The similarity between the Simplified SBP and the SPE advocated by Lewis holds as long as one considers the two setups in isolation. However, the difference in the absolute measures of existence of the alternative worlds in the two cases explains why the two setups cannot be treated as equivalent when used as a part of a larger experiment.

Our calculations are consistent with Peterson (2011), who, using Dutch book arguments, reached a thirder solution in the classical SBP, and a halfer solution in Lewis's double-coin setup. Peterson, however, does not question the validity of Lewis's modification of the QSBP.

Our method can be applied to other variations of SBP. For example, in Bradley's (2011) 'Technicolour' SBP, in addition to the original procedure, Beauty is shown a coloured flag upon each awakening. For Heads, Red or Blue are shown at random with equal probabilities. For Tails, Red is shown on Monday and Blue on Tuesday. Bradley argued that Beauty's credence in Heads should be $1 / 2$, independently of the colour she sees. Although we agree with Bradley that the credence in Heads is independent of the colour of the flag, we, like Alastair Wilson (2013), believe that Bradley is mistaken regarding the value of the credence.

To analyse this problem within the MWI, we introduce a second coin toss in the $H$-world (see Figure 3). Upon awakening, Beauty knows she is in one of four possible events: $H H_{M o n}(\operatorname{Red})$, $H T_{M o n}($ Blue $), T_{M o n}($ Red $)$ and $T_{T u e}($ Blue $)$. The measures of existence of the branches corresponding to $H H_{M o n}($ Red $)$ and $H T_{M o n}($ Blue $)$ are 1/4, while the branch associated with the other two events has measure $1 / 2$. Thus, upon awakening, but before Beauty sees the flag, her credence in Heads should be:

$$
C r(H)=\frac{\mu\left(H H_{\text {Mon }}\right)+\mu\left(H T_{\text {Mon }}\right)}{\mu\left(H H_{\text {Mon }}\right)+\mu\left(H T_{\text {Mon }}\right)+\mu\left(T_{\text {Mon }}\right)+\mu\left(T_{\text {Tue }}\right)}=\frac{\frac{1}{4}+\frac{1}{4}}{\frac{1}{4}+\frac{1}{4}+\frac{1}{2}+\frac{1}{2}}=\frac{1}{3}
$$

Once Beauty knows the colour, say, 'Red', the only viable options are the two Red awakenings:

$$
\operatorname{Cr}(H \mid R e d)=\frac{\mu\left(H H_{M o n}\right)}{\mu\left(H H_{M o n}\right)+\mu\left(T_{M o n}\right)}=\frac{\frac{1}{4}}{\frac{1}{4}+\frac{1}{2}}=\frac{1}{3}
$$


Seeing a Blue flag yields the same result. Thus, Beauty's credence in Heads in the Technicolour SBP before or after she sees the flag should be $1 / 3$, and not $1 / 2$ as argued by Bradley.

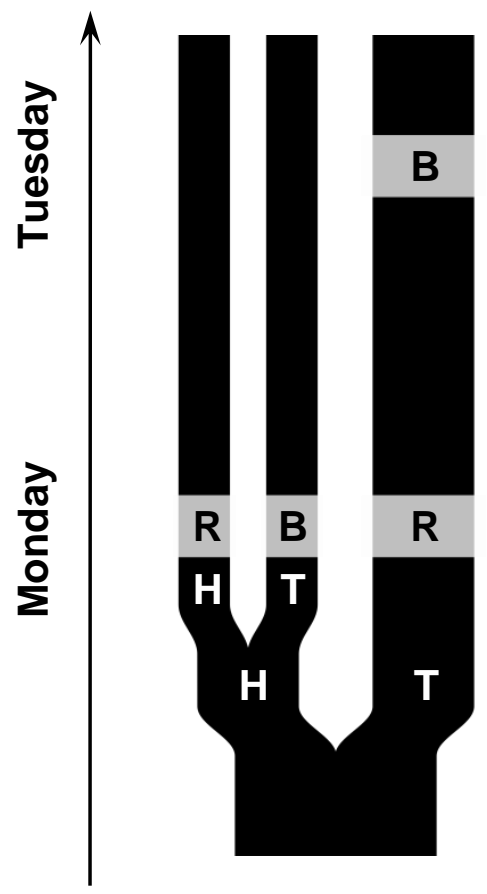

Figure 3: World lines of the 'Technicolour' Sleeping Beauty Problem. The measure of existence of the first coin $T$-branch is $1 / 2$, while those of the two $H$-branches are $1 / 4 . \mathrm{R}$ and $\mathrm{B}$ indicate the red and blue flag colours on the corresponding awakenings.

\section{The nature of a fair coin}

In the original SBP, Adam Elga and David Lewis did not discuss the nature of the fair coin. They considered it as a random variable with two equiprobable possible values, viewing the random variable as a primitive of classical probability theory. Vaidman (2001) used a quantum experiment as a fair coin, without analysing it in detail. Wilson (2013) suggested that the nature of the coin might matter (he distinguished between a 'random' coin and a 'mathematical' coin, see §7) and since a particular (quantum) implementation of the fair coin is crucial for our analysis, we have to justify Vaidman's move.

There is no place for a random variable in classical physics: knowing all the details about a system at a specific time, allows us to predict its future with certainty. In fact, it is standard quantum mechanics that can certify a genuine randomness via nonlocal correlations (Pironio et 
al. 2010). Without quantum mechanics or some hypothetical new theory which has chancy events in its foundation, we cannot toss a coin in a genuinely chancy way. However, we can implement a fair coin based on the ignorance of the agent. Instead of deciding upon the result of a coin toss on which we cannot rely, one party hides the coin in one of her hands and asks the other party to choose the hand.

In our experiment we use two identical boxes, $A$ and $B$, with a single ball hidden inside. On Sunday, Beauty marks the box which will be opened after she falls asleep. The researchers subsequently open the marked box. We define as 'Heads' the event of finding the ball in the marked box, otherwise - 'Tails'. Since Beauty herself chooses which box to mark without having any a priori knowledge about the location of the ball, this setup serves as a classical fair coin. A team of referees ensures that there is no less and no more than one ball in the boxes.

To implement a quantum coin, we use a quantum particle as the 'ball', and create the particle in a superposition of being in the two boxes, say, $\left|\Psi_{+}\right\rangle=\frac{1}{\sqrt{2}}(|\mathrm{~A}\rangle+|\mathrm{B}\rangle)$. The relative phase is not relevant so the state $\left|\Psi_{-}\right\rangle=\frac{1}{\sqrt{2}}(|\mathrm{~A}\rangle-|\mathrm{B}\rangle)$ is good too. In the framework of standard quantum mechanics, we have introduced randomness: no one knows the outcome of the quantum experiment even after Beauty had made her choice.

There are two separate issues to address. First, Beauty's credence does not change with the replacement of a classical coin by a quantum coin. Second, Beauty's belief in the MWI instead of the standard quantum mechanics does not change her credence regarding the outcomes of the quantum experiment.

Let us first address the second issue. There is a broad agreement among physicists that there is no current or foreseeable future technology which will allow one to perform an experiment distinguishing between the MWI and Collapse interpretations (Vaidman 2002). An observable change in Beauty's credence in the QSBP experiment would constitute an example of such an experiment and thus we find it unlikely. Moreover, in the framework of the decision theory, Greaves and Myrvold (2010) argued that a believer in the MWI should make the same rational decisions as an agent who believes in a Collapse theory. 
Let us now turn to the first issue. We will adopt here the standard approach in which every quantum measurement ends with a single outcome. We will also rely on a well-established physical principle according to which superluminal signaling is impossible. This will rule out an observable change in Beauty's credence, when the classical coin is replaced with a quantum coin. To prove our point we introduce a quantum state in which the location of the ball (box $A$ or $B$ ) is entangled with a spin-state of an ancillary spin-1/2 particle located in a faraway region:

$$
\left|\Psi_{E n t}\right\rangle=\frac{1}{\sqrt{2}}\left(|A\rangle\left|\uparrow_{z}\right\rangle+|B\rangle\left|\downarrow_{z}\right\rangle\right)
$$

If the faraway experimenter does not touch the spin particle, then it is again an experiment with quantum randomness. Moreover, measuring the spin in the $x$ direction will put the ball into one of the superpositions $\left|\Psi_{+}\right\rangle$or $\left|\Psi_{-}\right\rangle$:

$$
\left|\Psi_{E n t}\right\rangle=\frac{1}{2}|A\rangle\left(\left|\uparrow_{x}\right\rangle+\left|\downarrow_{x}\right\rangle\right)+\frac{1}{2}|B\rangle\left(\left|\uparrow_{x}\right\rangle-\left|\downarrow_{x}\right\rangle\right)=\frac{1}{\sqrt{2}}\left(\left|\uparrow_{x}\right\rangle\left|\Psi_{+}\right\rangle+\left|\downarrow_{x}\right\rangle\left|\Psi_{-}\right\rangle\right) \stackrel{\text { measurement }}{\longrightarrow}\left\{\begin{array}{l}
\left|\Psi_{+}\right\rangle \\
\left|\Psi_{-}\right\rangle
\end{array},\right.
$$

which leads to the quantum experiment described above. However, measuring the spin in the $z$ direction reduces the ball's state into one of the localized states $|A\rangle$ or $|B\rangle$, which leads to the classical coin experiment: After Beauty's choice the outcome of the coin toss is determined. Thus, an experimenter who performs a spin measurement in a faraway region, can instantaneously affect the nature of the Sleeping Beauty experiment: quantum or classical. So if there were a real difference between the two, he could send signals to Beauty faster than light!

\section{The operational meaning of Beauty's credence}

We have relied on the impossibility of superluminal signaling to rule out a 'real' difference in Beauty's credence between the quantum and classical cases. It cannot be just a semantic issue (as Groisman (2008) argued the difference between Adam Elga's and David Lewis approaches to be). Beauty's credence has to be observable.

Following de Finetti (1974), we identify credence with readiness to place a bet. Then we can test our theory by repeating the process many times and counting the amount of money Beauty gains (or loses). As has been noted many times before, when Beauty places bets on every awakening, 
she will be richer (on average) placing bets on Tails. It might seem that the rules of the game are unfair: Beauty gets advantage for Tails since she plays twice and thus gets a double amount of money in the case of Tails. Let us correct this by asking Beauty to place her bets only in one of the Tails awakenings. In this case she will not gain money with any strategy. This allegedly supports the halfer position.

Nevertheless, we still hold to our claim that on each awakening Beauty has credence one-third for Heads. If she herself could offer bets to a third party, she would do it according to a thirder approach. But when she is offered bets by the researcher who - as she knows - approaches her for every Heads, but only for half of Tails, she gets new information which changes her credence in Heads from $1 / 3$ to $1 / 2$.

\section{Mathematical Sleeping Beauty}

In Wilson's (2013) variant of the SBP - the Mathematical Sleeping Beauty (MSB) - the coin toss is replaced by Beauty's uncertainty regarding the truth of a mathematical proposition. Instead of marking the box, she chooses how the researchers should associate 'true' and 'false' with 'Heads' and 'Tails', which specify the awakenings pattern in the SBP. It is assumed that Beauty has no relevant mathematical training so that on Sunday she has credence $1 / 2$ for 'true' and $1 / 2$ for 'false'. Upon each awakening, Beauty is asked for her credence in the truth of the proposition.

While Wilson argued for the answer $1 / 3$ in the SBP, he advocated the halfer solution to the MSB. We, however, attribute a thirder solution to the MSB as well. We see no essential difference between the MSB and the SBP with a classical coin toss: in both cases there is a definite result that Beauty is not aware of. This uncertainty follows from either the lack of mathematical education, or her ignorance about the contents of the opaque boxes. Wilson built his argument on the difference between a classical chancy event and the definite truth value of a mathematical proposition. In our view, however, there are no chancy classical events: the result of any classical experiment is defined by the initial condition of the Universe. In any way, according to our analysis, for fair coins of all types only the thirder solution applies. 
We can apply our method also if Beauty were a mathematician and had a priori knowledge regarding the truth of the theorem, say, credence $p$ on Sunday in it being 'true'. Upon awakening, her credence should change to $\frac{p}{p+2(1-p)}=\frac{p}{2-p}$ in case she has chosen 'true' equals 'Heads', or to $\frac{2 p}{2 p+(1-p)}=\frac{2 p}{1+p}$ otherwise. Note that if Beauty is certain that the theorem is true (or false), her credence does not change.

Our analysis of the MSB helps to demonstrate the information transfer between the researchers and Beauty: While on Sunday Beauty is ignorant about the truth of the mathematical proposition, the researchers are not. Beauty understands that awakenings serve as evidence and thus, in the middle of the week, Beauty has more knowledge regarding the truth of the proposition. Similarly, in the original SBP she has more knowledge about the presence of a ball inside the box she marked.

\section{Conclusions}

We have demonstrated that the concept of the measure of existence of a world in the MWI provides an unambiguous solution to the QSBP. Contrary to the assertion of Peter Lewis, it is a thirder solution. We have argued that changing the nature of the coin cannot have any observable effects: in particular, there should not be a difference between Beauty's credences in the QSBP and the original classical SBP. Considering definite splitting of worlds in the MWI instead of uncertain outcomes provides a simple and powerful tool for analysing rational decision theory problems and, specifically, it can be applied to all setups discussed in the context of the SBP.

\section{Acknowledgements}

We are grateful to Jeremy Butterfield for very useful comments. This work was supported by Sidney Sussex College, Cambridge and by the Israel Science Foundation [grant number $1125 / 10]$. 


\section{References}

Bradley, D.J. 2011. Confirmation in a Branching World: The Everett Interpretation and Sleeping Beauty. The British Journal for the Philosophy of Science 62: 323-342.

de Finetti, B. 1974. Theory of Probability, trans. A. Machí and A. Smith, Vol. 1. New York: John Wiley \& Sons.

Elga, A. 2000. Self-locating belief and the Sleeping Beauty problem. Analysis 60: 143-147.

Greaves, H. and Myrvold, W. 2010. Everett and evidence. In Many Worlds?: Everett, Quantum Theory, and Reality, ed. S. Saunders, J. Barrett and A. Kent, 264-304. New York: Oxford University Press.

Groisman, B. 2008. The end of Sleeping Beauty's nightmare. The British Journal for the Philosophy of Science 59: 409-416.

Lewis, D. 2001. Sleeping Beauty: reply to Elga. Analysis 61: 171-176.

Lewis, P.J. 2007. Quantum Sleeping Beauty. Analysis 67: 59-65.

Lewis, P.J. 2009. Reply to Papineau and Durà-Vilà. Analysis 69: 86-89.

Papineau, D. and Durà-Vilà, V. 2009a. A thirder and an Everettian: a reply to Lewis's 'Quantum Sleeping Beauty'. Analysis 69: 78-86.

Papineau, D. and Durà-Vilà, V. 2009b. Reply to Lewis: metaphysics versus epistemology. Analysis 69: 89-91.

Peterson, D. 2011. Qeauty and the books: a response to Lewis's quantum Sleeping Beauty problem. Synthese 181: 367-374.

Pironio, S. et al. 2010. Random numbers certified by Bell's theorem. Nature 464: 1021-1024.

Pust, J. 2011. Sleeping Beauty and direct inference. Analysis 71: 290-293.

Saunders, S. and Wallace, D. 2008. Branching and uncertainty. The British Journal for the Philosophy of Science 59: 293-305. 
Tappenden, P. 2011. Evidence and uncertainty in Everett's multiverse. The British Journal for the Philosophy of Science 62: 99-123.

Thorn, P.D. 2011. Undercutting defeat via reference properties of differing arity: a reply to Pust. Analysis 71: 662-667.

Vaidman, L. 1998. On schizophrenic experiences of the neutron or why we should believe in the many-worlds interpretation of quantum theory. International Studies in the Philosophy of Science 12: 245-261.

Vaidman, L. 2001. Probability and the many worlds interpretation of quantum theory. In Quantum theory: Reconsideration of foundations, ed. A. Khrennikov, 408-412. Sweden: Vaxjo University Press.

Vaidman, L. 2002. Many-worlds interpretation of quantum mechanics. In The Stanford Encyclopedia of Philosophy, ed. E. Zalta. http://plato.stanford.edu/archives/sum2002/entries/qmmanyworlds/Vaidman, L. 2012. Probability in the many-worlds interpretation of quantum mechanics. In Probability in physics, ed. Y. Ben-Menahem and M. Hemmo, 299-311. Berlin Heidelberg: Springer.

Wilson, A. 2013. Everettian confirmation and Sleeping Beauty. Forthcoming in the British Journal for the Philosophy of Science, http://alastairwilson.org/files/ecsbweb.pdf. 